\title{
Implementation of Novel Biomarkers in the Diagnosis, Prognosis, and Management of Acute Kidney Injury: Executive Summary from the Tenth Consensus Conference of the Acute Dialysis Quality Initiative (ADQI)
}

Peter A. McCullough ${ }^{1} \cdot$ Josee Bouchard ${ }^{2}$ - Sushrut S. Waikar ${ }^{3}$. Edward D. Siew ${ }^{4} \cdot$ Zoltan H. Endre ${ }^{5}$ Stuart L. Goldstein ${ }^{6}$. Jay L. Koyner ${ }^{7} \cdot$ Etienne Macedo ${ }^{8} \cdot$ Kent Doi $^{9} \cdot$ Salvatore Di Somma ${ }^{10}$. Andrew Lewington ${ }^{11} \cdot$ Ravi Thadhani ${ }^{12}$. Raj Chakravarthi ${ }^{13} \cdot$ Can Ice $^{14}$ - Mark D. Okusa ${ }^{15}$. Jacques Duranteau ${ }^{16} \cdot$ Peter Doran ${ }^{17} \cdot$ Li Yang $^{18}$. Bertrand L. Jaber ${ }^{19}$ • Shane Meehan 20 • John A. Kellum²1 . Michael Haase 22 . Patrick T. Murray ${ }^{23}$ • Dinna Cruz ${ }^{24}$. Alan Maise $^{25} \cdot$ Sean M. Bagshaw ${ }^{26}$ • Lakhmir S. Chawla 27 . Ravindra L. Mehta ${ }^{28} \cdot$ Andrew D. Shaw ${ }^{29} \cdot$ Claudio Ronco ${ }^{30} \cdot$ for the Acute Dialysis Quality Initiative (ADQI) Consensus Group

${ }^{1}$ St. John Providence Health System, Warren, Mich., Providence Hospitals and Medical Centers, Southfield and Novi, Mich., St. John Macomb Oakland Center, Madison Heights, Mich., St. John Hospital and Medical Center, Detroit, Mich., USA; ${ }^{2}$ Division of Nephrology and Hypertension, Division of Nephrology, Université de Montréal, Montréal, Que., Canada; ${ }^{3}$ Renal Division, Department of Medicine, Brigham and Women's Hospital, Harvard Medical School, Boston, Mass., USA; ${ }^{4}$ Division of Nephrology and Hypertension, Department of Medicine, Vanderbilt University School of Medicine, Nashville, Tenn., USA; ${ }^{5}$ Christchurch Kidney Research Group, Department of Medicine, University of Otago, Christchurch, New Zealand; ${ }^{6}$ Center for Acute Care Nephrology, Division of Nephrology and Hypertension, The Heart Institute, Cincinnati Children's Hospital Medical Center, Cincinnati, Ohio, USA; ${ }^{7}$ Section of Nephrology, Department of Medicine, University of Chicago, Pritzker School of Medicine, Chicago, III., USA; ${ }^{8}$ Division of Nephrology, University of São Paulo, São Paulo, Brazil; ${ }^{9}$ Department of Nephrology and Endocrinology, The University of Tokyo, Tokyo, Japan; ${ }^{10}$ Sant'Andrea Hospital, Second Faculty Medical School, La Sapienza University of Rome, Rome, Italy; ${ }^{11}$ Leeds Teaching Hospitals, Leeds, UK; ${ }^{12}$ Division of Nephrology, Massachusetts General Hospital, Boston, Mass., USA; ${ }^{13}$ Division of Nephrology, Albany Medical College, Albany, N.Y., USA; ${ }^{14}$ Department of Intensive Care Adults, Erasmus MC University Medical Centre Rotterdam, 
Rotterdam, The Netherlands; ${ }^{15}$ University of Virginia School of Medicine, Charlottesville, Va., USA;

${ }^{16}$ Nephrology Unit, Le Kremlin Bicetre, Bicetre, France; ${ }^{17}$ Medizinische Poliklinik, University of Munich, Munich, Germany; ${ }^{18}$ Renal Division, Brigham and Women's Hospital, Boston, Mass., USA;

${ }^{19}$ Division of Nephrology, Tufts Medical Center, Boston, Mass., USA; ${ }^{20}$ Department of Pathology, Section of Nephrology, University of Chicago, Chicago, III., USA; ${ }^{21}$ Clinical Research, Investigation, and Systems Modeling of Acute Illness Center, Department of Critical Care Medicine, University of Pittsburgh, Pittsburgh, Pa., USA; ${ }^{22}$ Department of Nephrology, Hypertension, Diabetes and Endocrinology, Otto von Guericke University, Magdeburg, Germany; ${ }^{23}$ School of Medicine and Medical Science, University College Dublin, Dublin, Ireland; ${ }^{24}$ Department of Nephrology Dialysis and Transplantation, San Bortolo Hospital, Vicenza, Italy; ${ }^{25}$ Department of Medicine and Cardiology, San Diego VA Medical Center and University of California, San Diego, Calif., USA; ${ }^{26}$ Division of Critical Care Medicine, Faculty of Medicine and Dentistry, University of Alberta, Edmonton, Alta., Canada;

${ }^{27}$ Department of Anesthesiology and Critical Care Medicine and Division of Renal Diseases and Hypertension, Department of Medicine, George Washington University Medical Center, Washington, D.C., USA; ${ }^{28}$ Division of Nephrology and Hypertension, Department of Medicine, University of California San Diego, San Diego, Calif., USA; ${ }^{29}$ Department of Anesthesiology, Duke University Medical Center, Durham, N.C., USA; ${ }^{30}$ Department of Nephrology, International Renal Research Institute (IRRIV), San Bortolo Hospital, Vicenza, Italy

\begin{abstract}
Detection of acute kidney injury is undergoing a dynamic revolution of biomarker technology allowing greater, earlier, and more accurate determination of diagnosis, prognosis, and with powerful implication for management. Biomarkers can be broadly considered as any measurable biologic entity or process that allows differentiation between normal function and injury or disease. The ADQI (Acute Dialysis Quality Initiative) had its Ninth Consensus Conference dedicated to synthesis and formulation of the existing literature on biomarkers for the detection of acute kidney injury in a variety of settings. In the papers that accompany this summary, ADQI workgroups fully develop key concepts from a summary of the literature in the domains of early diagnosis, differential diagnosis, prognosis and management, and concurrent physiologic and imaging measures.
\end{abstract}

Copyright $\odot 2013$ S. Karger AG, Basel

Acute kidney injury (AKI) is increasingly recognized as an in-hospital complication that is common and strongly related to increased hospital length of stay, use of intensive care unit services including hemodynamic monitoring, need for renal replacement therapy, rehospitalization, and death. When patients are followed after an episode of AKI, there are higher risks for the rapid progression of chronic kidney disease leading to end-stage renal disease requiring permanent dialysis therapy or renal transplantation. Hence, there is a great need to better understand AKI with the intent of developing prevention and treatment strategies. Prior to the past few years, the principle clinical tools used to detect AKI were serial measurement of serum creatinine (Cr), blood urea nitrogen, assessment of the urinalysis, and measurement of urine output. Agreement from multiple consensus groups (Acute Kidney Injury Network - AKIN, Risk, Injury, Failure, End-Stage Kidney Disease - RIFLE, and Kidney Disease Improving Global Outcomes - 


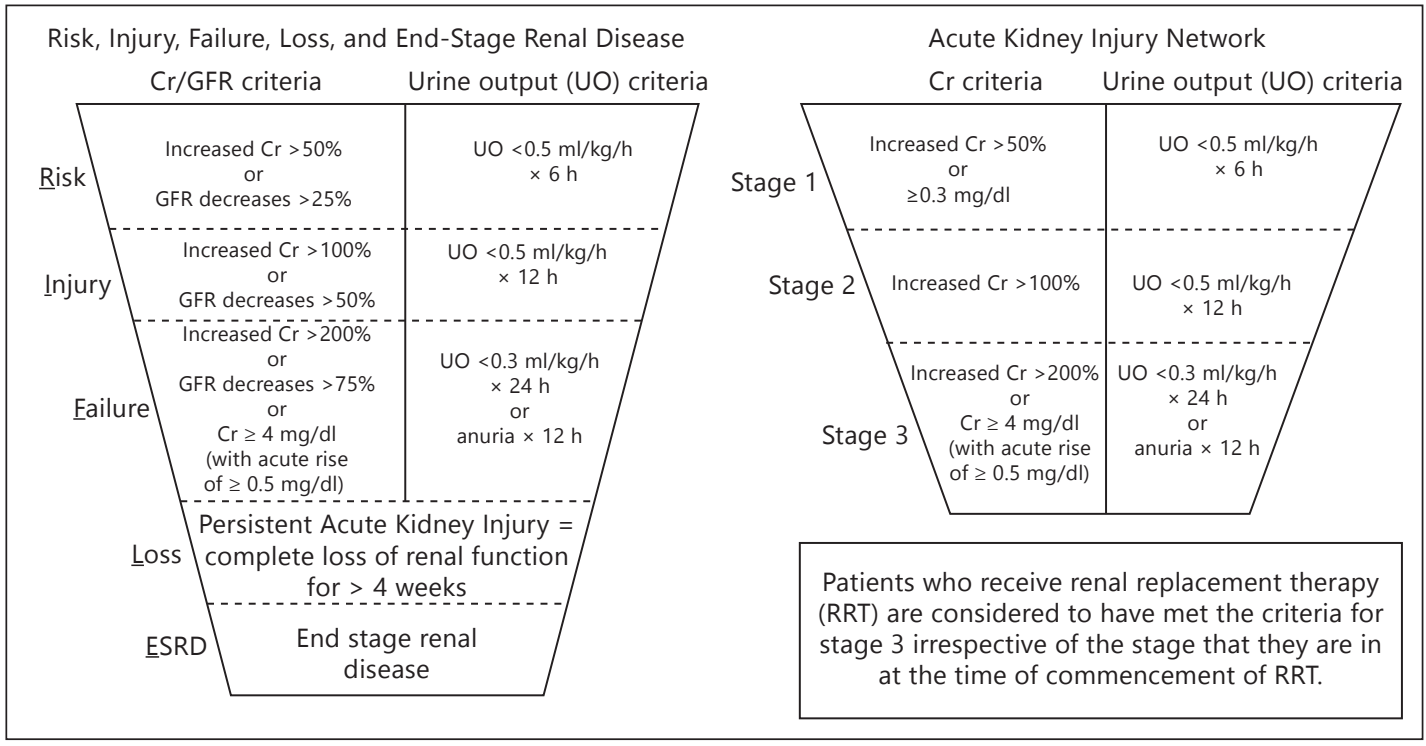

Fig. 1. The AKIN and RIFLE definitions and grading for AKI.

KDIGO) that AKI can be defined and graded has been a major advance in the phenotypic characterization as shown in figure 1 [1]. Thus the stage has been set for new technologies and measures to be assessed in the context of both the diagnosis and grading of AKI according to these standardized definitions [2].

\section{Methods}

The ADQI process was applied using previously described methodology taking advantage of key opinion leaders in the field and identifying appropriate topics for consensus [3]. The ADQI methodology comprises a systematic search for evidence with review and evaluation of relevant literature, establishment of clinical and physiologic outcomes for comparison of different treatments, description of current practice and analysis of areas in which evidence is lacking and a specific research agenda is required. ADQI activities were divided into a pre-conference, conference, and post-conference phase. Before the conference, topics were selected and workgroups assembled. Groups identified key questions and conducted a systematic literature search. During the conference, workgroups assembled in breakout sessions, as well as plenary sessions where their findings were presented, debated, and refined. Key questions were identified by the entire ADQI group, and the subgroups deliberated on these questions, bringing forth recommendations to the group as a whole. Deliberations followed three days of discussion amongst the attendees. Summary statements were then developed by the entire group and reported into the present document. 


\section{Results}

\section{Early Detection of Acute Kidney Injury}

The first ADQI workgroup focused on the available literature on novel blood and urine biomarkers that have been tested in clinical studies for the early detection of AKI. Recognition that cells in the kidney produce and release measurable proteins in the setting of a variety of injuries has been an important step in the development of this field [4]. Injury appears to be as subtle as volume depletion which has been previously termed 'prerenal azotemia'. Data with novel markers suggest these episodes are indeed a form of AKI with some damage to cells and or complete nephron units [5]. More severe causes of renal damage include prolonged ischemia due to shock, sepsis and microcirculatory dysfunction, and direct toxicity due to injurious agents such as iodinated contrast, aminoglycosides, and vancomycin. Most of the markers mentioned in the accompanying article by McCullough and colleagues are either directly produced by renal tubular cells in response to upregulation of messenger RNA and protein synthesis, or are filtered proteins that have reduced reabsorption by the proximal tubular cells, and hence become measurable in greater quantities in the urine. Since the completion of the ADQI 10 meeting, and important discovery that tubular cell-cycle arrest markers (insulin-like growth factor-binding protein 7 (IGFBP7) and tissue inhibitor of metalloproteinases-2 (TIMP-2)) appear to be a brief ( $36 \mathrm{~h}$ ) harbinger to AKI that will develop in the next few days [6]. The concentrations of these proteins in the urine can be multiplied to create a value for use by clinicians to anticipate the immediate risk of AKI. The development of AKI from initiation of injury forward appears to be reflected by sharp elevation of neutrophil gelatinase-associated lipocalin (NGAL), kidney injury molecule-1 (KIM-1), L-type fatty acid-binding protein (L-FABP), and $\alpha$-glutathione S-transferase ( $\alpha$-GST), $\pi$-GST, and interleukin-18 (IL-18). The preponderance of the data suggests these novel markers detect risk for AKI and probably actual AKI 24-48 h before the rise in serum Cr [7]. Importantly, there are cases where novel markers significantly elevate without a significant change in serum $\mathrm{Cr}$ or reduction in urine output, which is a late and ominous clinical development [8]. These cases of subclinical AKI appear to have important prognostic implications and probably should not be dismissed (fig. 2).

\section{Differential Diagnosis}

One of the most important uses of novel biomarkers is to help evaluate a patient who has a differential diagnosis in the setting of critical illness including pulmonary, heart, liver, and kidney failure. The novel markers in 


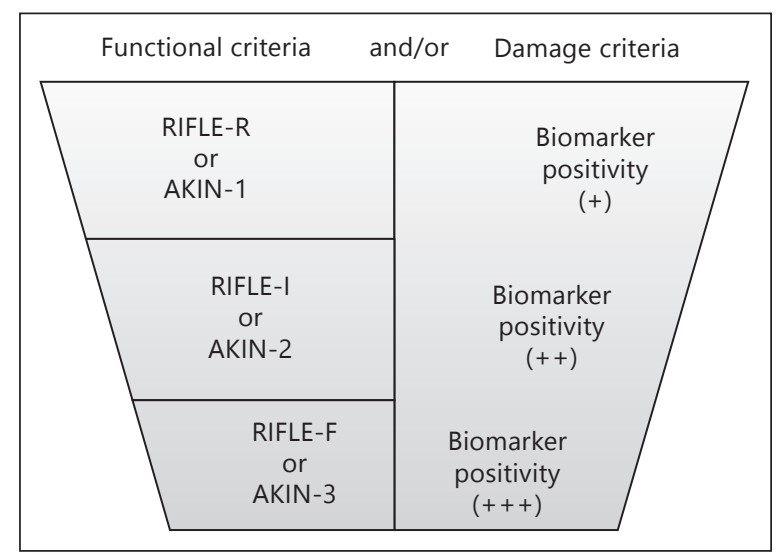

Fig. 2. Depiction of damage and functional markers in the detection of AKI. Reproduced with permission from ADQI [15].

aggregate appear to have moderate specificity for AKI. Some forms of injury, particularly those with abnormalities in cell signaling (cytokines, adhesion molecules) and microvascular dysfunction, appear to favor interleukins (IL18), NGAL, and KIM-1 as relatively strong signals for AKI due to sepsis [9]. For most forms of injury, urinary levels of these markers are higher in concentration and have a greater rise than the same proteins measured in blood. When there are increases in urinary cystatin $\mathrm{C}$ and albumin from baseline, this suggests a failure in proximal renal tubular reabsorption [10]. This finding is complementary to information provided by the novel biomarkers above, particularly in establishing AKI in the differential diagnosis of the critically ill patient.

\section{Prognosis and Management}

Because AKI is strongly associated with early and late mortality, even when renal replacement therapy is offered, biomarkers indicating AKI are prognostic for mortality. In general, the greater the rise in a single marker, more consistent elevations of more than one marker, and when in conjunction with a reduction in glomerular filtration and urine output, there is a quantitative relationship between the severity of AKI and clinical outcomes. Thus, as shown in figure 3, with more prognostic information at an earlier stage of critical illness, there can be a series of clinical responses leading to reduced complications and possibly improvement in outcomes [1]. The underlying principles have now emerged: (1) novel markers allow early assessment of prognosis, (2) advanced warning can 


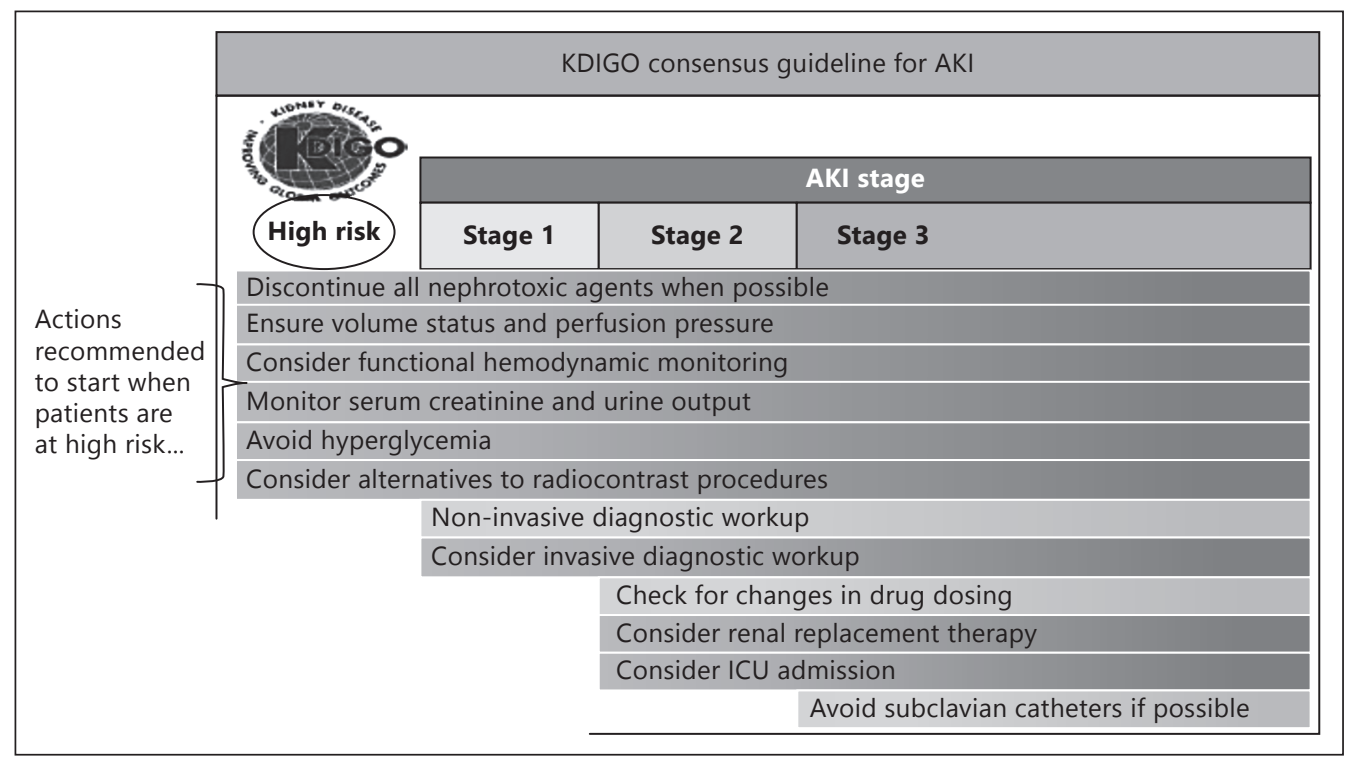

Fig. 3. Recognition of AKI and clinical responses. Adapted from KDIGO [1].

lead to changes in clinical processes, and (3) an alerted clinician and care team can more effectively care and communicate with patients and their families leading to improvements in delivery [11].

\section{Physiologic and Imaging Measures}

Cardiovascular medicine and oncology have taught us that the combination of laboratory measures and imaging/physiological assessments are powerful in terms of establishing internal validity, disease definitions, staging, and monitoring of progression of disease. Thus, in AKI, considerable work has been undertaken to understand core features of renal function including renal blood flow, regional renal perfusion, metabolism of glucose and other substrates, and the net control over renal filtration and urine output. A current standard of care in a case of de novo AKI includes renal ultrasonography which primarily establishes the presence of polycystic kidney disease, ureteral disease and obstruction, and helps somewhat to establish or support baseline chronic kidney disease with measurement of renal size. Ultrasound, however, is not helpful in diagnosing AKI in patient with normal size kidneys and no obstruction to urinary flow. The most promising novel approaches include renal time assessment of renal blood flow, oxygenation, and glomerular filtration $[12,13]$. It is possible that discovery 
of a drop in renal filtration could occur many hours/days before serum Cr elevates, and thus give useful information to the clinician following the management principles discussed above [14].

\section{Conclusions}

$\mathrm{AKI}$ is a dynamic process that generates a multitude of biologic signals that when measured, become clinical biomarkers. Use of these markers appears to be valuable in the detection, differential diagnosis, prognosis, and management of patients in the setting of critical illness. Future research focused on the development and use of new diagnostic and therapeutic targets is critical to advance the field of critical care nephrology.

\section{Acknowledgements}

The ADQI 10 workgroup members are indebted to the independent monitoring of the ADQI process provided by the individuals listed below. These professionals provided content information and resources to the groups, but had no role in the writing or review of this or any of the ADQI 10 articles.

Funding Source: The 9th International ADQI Consensus Conference was funded in part by generous support in form of unrestricted educational grants from Alere Inc., and Astute Medical Inc. The sponsors had no input on the content of this paper.

Special Rotating Observers: Group A: Paul Clopton (USA), Joe Bonventre (USA); Group B: Paul Kimmel (USA), Bruce Molitoris (USA); Group C: Prasad Devarajan (USA), Maria Fitzgibbon (Ireland), and Group D: Eisei Noiri (Japan), Chirag Parikh (USA).

\section{References}

1 KDIGO AKI Guidelines: Summary of recommendation statements. Kidney Int Suppl 2012;2:8-12.

-2 Uchino S, Bellomo R, Goldsmith D, Bates S, Ronco C: An assessment of the RIFLE criteria for acute renal failure in hospitalized patients. Crit Care Med 2006;34:1913-1917.

$\checkmark 3$ Kellum JA, Bellomo R, Ronco C: Acute Dialysis Quality Initiative (ADQI): methodology. Int J Artif Organs 2008;31:90-93.
-4 Boudonck KJ, Rose DJ, Karoly ED, Lee DP, Lawton KA, Lapinskas PJ: Metabolomics for early detection of drug-induced kidney injury: review of the current status. Bioanalysis 2009;1:1645-1663.

5 McCullough PA, Chinnaiyan KM, Gallagher MJ, Colar JM, Geddes T, Gold JM, Trivax JE: Changes in renal markers and acute kidney injury after marathon running. Nephrology (Carlton) 2011;16:194-199.

6 Kashani K, Al-Khafaji A, Ardiles T, et al: Discovery and validation of cell cycle arrest biomarkers in human acute kidney injury. Critical Care 2013; 17:R25. 
7 Doi K, Negishi K, Ishizu T, Katagiri D, Fujita T, Matsubara T, et al: Evaluation of new acute kidney injury biomarkers in a mixed intensive care unit. Crit Care Med 2011;39:24642469.

$>8$ Prowle JR, Liu YL, Licari E, Bagshaw SM, Egi M, Haase M, et al: Oliguria as predictive biomarker of acute kidney injury in critically ill patients. Crit Care 2011;15:R172.

-9 Srisawat N, Kellum JA: Acute kidney injury: definition, epidemiology, and outcome. Curr Opin Crit Care 2011;17:548-555.

10 Urbschat A, Obermüller N, Haferkamp A: Biomarkers of kidney injury. Biomarkers 2011;16(suppl 1):S22-S30.

11 McCullough PA: Multimodality prevention of contrast-induced acute kidney injury. Am J Kidney Dis 2008;51:169-172.
12 Liu PS, Platt JF: CT angiography of the renal circulation. Radiol Clin North Am 2010;48: 347-365, viii-ix.

13 Inoue T, Matsuoka H, Higashi Y, Ueda S, Sata M, Shimada KE, Ishibashi Y, Node K, Vascular Failure Workshop Group: Flowmediated vasodilation as a diagnostic modality for vascular failure. Hypertens Res 2008; 31:2105-2113.

14 Wang E, Meier DJ, Sandoval RM, Von Hendy-Willson VE, Pressler BM, Bunch RM, Alloosh M, Sturek MS, Schwartz GJ, Molitoris BA: A portable fiberoptic ratiometric fluorescence analyzer provides rapid point-of-care determination of glomerular filtration rate in large animals. Kidney Int 2012;81:112-117.

15 Acute Dialysis Quality Initiative. www.ADQI. org (accessed January 10, 2013).

Peter A. McCullough, MD, MPH

Providence Park Heart Institute, Providence Park Hospital

47601 Grand River, B125

Novi, MI 48374 (USA)

E-Mail peteramccullough@gmail.com 